\title{
Correction to: Arthroscopic correction of femoroacetabular impingement improves athletic performance in male athletes
}

\author{
Karen Mullins ${ }^{1}\left[\right.$ D $\cdot$ Michael Hanlon ${ }^{2} \cdot$ Patrick Carton $^{2,3}$
}

Published online: 24 October 2019

(c) European Society of Sports Traumatology, Knee Surgery, Arthroscopy (ESSKA) 2019

\section{Correction to: \\ Knee Surgery, Sports Traumatology, Arthroscopy \\ https://doi.org/10.1007/s00167-019-05683-0}

Unfortunately, the email of author "Patrick Carton" was incorrectly updated in the original publication and correct version is updated here.

Publisher's Note Springer Nature remains neutral with regard to jurisdictional claims in published maps and institutional affiliations.

The original article can be found online at https://doi.org/10.1007/ s00167-019-05683-0.

Karen Mullins

Karen.Mullins@lit.ie

Michael Hanlon

mhanlon@wit.ie

Patrick Carton

pcarton.secretary@whitfieldclinic.ie

1 Department of Sports Leisure and Tourism, Limerick Institute of Technology, Moylish Park, Limerick, Ireland

2 Department of Health Sport and Exercise Science, Waterford Institute of Technology, Co Waterford, Ireland

3 The Hip and Groin Clinic, UPMC Whitfield, Co Waterford, Ireland 